\title{
On the Injectivity of an Integral Operator Connected to Riemann Hypothesis
}

Dumitru Adam ( $\nabla$ dumitru_adam@yahoo.ca )

\section{Research Article}

Keywords: Approximation Subspaces, Linear Operators, Riemann Hypothesis

Posted Date: November 17th, 2022

DOI: https://doi.org/10.21203/rs.3.rs-1159792/v9

License: (9) (i) This work is licensed under a Creative Commons Attribution 4.0 International License. Read Full License 


\title{
On the Injectivity of an Integral Operator Connected to Riemann Hypothesis
}

\author{
Dumitru Adam*
}

${ }^{*}$ No affiliations. No conflict of interests, no funding. Ex-Researcher at Former Dept. of Math. INCREST \& Inst. of Math. Romanian Acad., Bucharest, Romania. Ex-Lecturer at Catholic Univ., Milan, Italy.

Addr. Windsor, On, N9A 3A5, Ca \& Brasov, Ro.dumitru_adam@yahoo.ca

\begin{abstract}
This version is a straightforward and more intuitive approach, pointing out the logic behind the method $([5],[6])$ used to investigate the injectivity of linear operators on separable Hilbert spaces. On a dense family of including finite dimension subspaces, we highlighted the connection between the projections of the elements outside the dense family and the condition numbers of the linear operator restrictions to the family subspaces. The connection we established is: for a zero of unitary norm of an operator linear strict positive definite on the dense family, on each subspace the ratio between its norms of the residuum and the corresponding projection is majorizing the condition number of the operator restriction. So, we proved that a criteria for injectivity is the existence of a strict positive inferior bound for the sequence of the condition numbers associated to the operator restrictions on the subspaces.

To address the Alcantara-Bode equivalent formulation ([1]) of the Riemann Hypothesis using the criteria, we choose a suitable dense family of subspaces on the separable Hilbert space $\mathrm{L}^{2}(0,1)$ well known in literature built on indicator interval functions with disjoint support. Numerically, we obtained a 'multigrid' structure of the finite dimension including approximation subspaces that together with the orthogonal projection schema defined in [3] are fitting the requests of the method introduced.
\end{abstract}

As result we obtained ([5], [6]): the integral operator used by AlcantaraBode in its equivalent formulation of $\mathrm{RH}$ ([1]) has the condition numbers sequence bounded by a constant, showing that the operator used in this equivalence is injective.

Then, we could claim: the Riemann Hypothesis holds provided that its equivalent formulation is true.

Keywords: Approximation Subspaces, Linear Operators, Riemann Hypothesis

Subjclass: 65R99, 47G10, 45P05 
1. Introduction. Let $\mathrm{T}$ be an operator linear on a separable Hilbert space $\mathrm{H}$, having a not null zero $(\mathrm{Tu}=0)$. If there exists a subspace $\mathrm{S}$ on which $\mathrm{T}$ is strict positive definite, then the zero is not in the subspace $\mathrm{S}$ otherwise the inner product $\langle\mathrm{Tu}, \mathrm{u}\rangle=0$ is violating the strict positivity of $\mathrm{T}$ on $\mathrm{S}$. If $\mathrm{T}$ is strict positive definite on any subspace of $\mathrm{H}$ then it is injective.

Investigating the injectivity of a linear operator, this approach is not productive from a practical point of view. Our choice consists in:

(a). Choose a dense family of finite dimension including subspaces and take the difference sets between the whole space and the union of the subspaces of the dense family. If an linear operator strict positive definite on every subspace of the family has a zero, then the zero should be in the difference sets. Moreover, we'll select from this difference sets only the elements of norm 1, that will ease our proofs - these elements are the 'eligible' elements to be zeros of the operators linear and strict positive definite on the family.

(b). Then, in order to investigate the injectivity of an operator linear strict positive definite on a dense family we have to define a criteria involving the eligible set and the operator restrictions on the subspaces of that family.

Some elementary observations are needed before starting:

- $\mathrm{T}$ and $\mathrm{T}^{*} \mathrm{~T}$ - its associated Hermitian, have both the same null space; so, we could switch the investigation to $\mathrm{T}^{*} \mathrm{~T}$ when no information about the positivity of $\mathrm{T}$ there exists.

- $\mathrm{u}$ (not null) and $\mathrm{u} /\|u\|$ both are, or are not zeros of $\mathrm{T}$. So, taking in consideration only the normalized potential zeros is not restricting the generality.

Let $\mathrm{F}=\left\{S_{n} ; n \geq 1\right\}$ be a dense family of including subspaces in $\mathrm{H}$ verifying: $S_{n} \subset S_{n+1}$ and $\overline{\left.\cup_{n=1, \infty} S_{n}\right)}=\mathrm{H}$. We denote with $\mathrm{L}_{F}$ the class of the linear operators strict positive definite on every subspace of $\mathrm{F}$.

If $\mathrm{T} \in \mathrm{L}_{F}$ then for every $\mathrm{S}_{n} \in F$ there exists $\alpha_{n}>0$ such that $\langle T v, v\rangle \geq$ $\alpha_{n}\|v\|^{2} \forall v \in \mathrm{S}_{n}$.

Let $\omega_{n}$ be verifying $\left\|T^{*} v\right\| \leq \omega_{n}\|v\|, \forall \mathrm{v} \in \mathrm{S}_{n}, \mathrm{n} \geq 1$.

Taking $\mathrm{B}:=\{u \in H,\|u\|=1\}$ and $\mathrm{E}:=\left(\mathrm{H} \backslash\left(\cup_{n=1, \infty} S_{n}\right)\right) \cap \mathrm{B}$, for $\mathrm{u} \in \mathrm{E}$ we define:

- $\forall u \in \mathrm{H}$ and $\forall n \geq 1, \mathrm{u}_{n}$ denotes the orthogonal projection of $\mathrm{u}$ on the subspace $\mathrm{S}_{n} \in \mathrm{F}$ and $n_{0}:=n_{0}(u)$, is the index from where $u_{n} \neq 0$.

- The condition number of an linear operator $\mathrm{T} \in L_{F}$ on a subspace $\mathrm{S}_{n}$, $\mu_{n}(\mathrm{~T})$ is the ratio between its strict positivity parameter $\alpha_{n}$ and $\omega_{n}$, the norm of the restriction of its adjoint; when $\mathrm{T}=\mathrm{T}^{*}$ then $\omega_{n}=\lambda_{\max }\left(\left.\mathrm{T}\right|_{S_{n}}\right)$ obtaining $\mu_{n}(\mathrm{~T})=\lambda_{\min }\left(\left.\mathrm{T}\right|_{S_{n}}\right) / \lambda_{\max }\left(\left.\mathrm{T}\right|_{S_{n}}\right)([5],[6])$. 
2. The Criteria. From now on, $\mathrm{T} \in L_{F}$.

Lemma 1. If $\mathrm{u} \in N_{T} \cap B$ then $\mathrm{u} \in \mathrm{E}$ and,

(1) $\quad \mu_{n} \leq \varepsilon_{n}(u) \quad \forall \mathrm{n} \geq n_{0}:=n_{0}(u)$

with $\mu_{n}=\alpha_{n} / \omega_{n}$ and $\varepsilon_{n}(u)=\beta_{n}(u) /\left\|u_{n}\right\|=\beta_{n}(u) / \sqrt{1-\beta_{n}^{2}(u)}$.

Proof. If $\mathrm{u} \notin E$ then $\mathrm{T} \notin L_{F}$ that is a contradiction. If $\mathrm{u} \in N_{T} \cap B$ then $\forall v \in \mathrm{H}, \mathrm{Tv}=\mathrm{T}(\mathrm{v}-\mathrm{u})$. On every $\mathrm{S}_{n}, \mathrm{n} \geq n_{0}$ taking $\mathrm{v}=\mathrm{u}_{n}$ holds:

$\alpha_{n}\left\|u_{n}\right\|^{2} \leq\left\langle T\left(u_{n}-u\right), u_{n}\right\rangle=\left\langle\left(u_{n}-u\right), T^{*} u_{n}\right\rangle \leq \omega_{n} \beta_{n}(u)\left\|u_{n}\right\|$ proving (1).

Let observe that for every $\mathrm{u} \in E,\left\|u_{n}\right\| \rightarrow 1, \beta_{n}(u) \rightarrow 0$ and $1=\|u\|^{2}=$ $\left\|u_{n}\right\|^{2}+\beta_{n}(u)^{2}$. Follows $\varepsilon_{n}(u) \rightarrow 0$ for $n \rightarrow \infty$.

Moreover, if there exists $\mathrm{u} \in E$ such that

$$
\mu_{n}>\varepsilon_{n}(u) \quad \forall \mathrm{n} \geq n_{0}:=n_{0}(u) \text { then } \mathrm{u} \notin N_{T} .
$$

Observation: if (2) holds for every $\mathrm{u} \in E$ then $\mathrm{T}$ is injective.

So, a criteria for injectivity could be as follows.

Theorem 1 - Injectivity Criteria. Let $\mathrm{T} \in L_{F}$. If there exists a constant $\mathrm{C}>0$ such that $\mu_{n}>C$ for every $\mathrm{n} \geq 1$, then $\mathrm{T}$ is injective.

Proof. Suppose $\mu_{n}>C>0$ for every $\mathrm{n} \geq 1$. Now, for $\mathrm{u} \in \mathrm{E}$ there exists $\mathrm{n}_{1}(u)$ such that for $\mathrm{n} \geq n_{1}(u)$ the following inequality holds: $\mathrm{C} \geq \varepsilon_{n}(u)$. Thus (2) holds proving $\mathrm{u} \notin N_{T} ; \mathrm{u}$ being arbitrary, $\mathrm{T}$ is injective on $\mathrm{H}$.

3. A dense family for $\mathbf{L}^{2}$ spaces. Let $H:=L^{2}(0,1)$.

A suitable dense family of approximation subspaces $\mathrm{F}:=\chi$, is given by subspaces generated by indicator interval functions having disjoint supports of equal length of mesh $\mathrm{h}, \mathrm{nh}=1$.

On a such subspace $\mathrm{S}_{h}:=\operatorname{span}\left\{\chi_{h, k}, k=1, n, n h=1\right\} \in \chi$, where $\chi_{h, k}(x)=1$ for $x \in \Delta_{k}:=((k-1) h, k h]$ and 0 for $\mathrm{x}$ outside of $\Delta_{k}$ the elements are simple functions, linear combinations of the indicator interval (orthogonal) functions generating the subspace. The family of the orthogonal indicator interval functions $\left\{\chi_{h, k}, k=1, n, n h=1\right\}$ defines a trace class integral operator that is an orthogonal projection in $\mathrm{H}$ over $\mathrm{S}_{h}([3])$.

If $\varphi$ is the kernel function of the integral operator $T_{\varphi}$ then its approximation on $\mathrm{S}_{h}$ is (see [3]):

(3) $\varphi_{h}(y, x)=h^{-1} \sum_{k=1, n} \chi_{h, k}(y) \varphi(y, x) \chi_{h, k}(x)$

Thus, we have in $\mathrm{L}^{2}(0,1)$ the dense family of finite dimension including subspaces halving the length of the support of the indicator interval functions, from a subspace to the next one.

From numerical analysis point of view, we just obtained a multi-grid discretization schema on which the matrix representations of a linear operator are sparse matrices 1-diagonal due to the disjoint support of the set of indicator interval functions spanning the finite dimension subspaces, i.e. $\mathrm{M}_{h}=\left[a_{i j}^{h}\right]_{i, j=1, n}, n h=1$ where $a_{i j}^{h}:=\left\langle T_{\varphi_{h}} \chi_{h, i}, \chi_{h, j}\right\rangle=0$ for $\mathrm{i} \neq j$. 
Regarding the density of the family of subspaces see [4] where is proved: (pg. 69, theorem 3.13.) the collection of all simple functions is dense in $\mathrm{L}^{p}$ spaces. As a particular case, the linear combinations of the functions $\chi_{h, k}$, $\mathrm{k}=1, \mathrm{n}, \mathrm{nh}=1$, spanning $\mathrm{S}_{h}$, with $\mathrm{S}_{h} \subset \mathrm{S}_{h / 2}, \mathrm{n} \rightarrow \infty$, is a dense collection of simple functions in $\mathrm{L}^{2}(0,1)$. (See also the very simple responses on this matter in math. stackexchange. com, to questions $808549,4265006,1883191$ )

4. On the Alcantara-Bode equivalence of RH. The kernel function $\varphi=\{y / x\}$ where the braces define the fractional part of the quantity inside them, is Lebesgue integrable, discontinue on a set of measure Lebesgue zero and bounded, $0 \leq \varphi(y, x)<1$ for every $\mathrm{x}, \mathrm{y}$ in $(0,1)$. The Alcantara-Bode equivalent formulation of RH (Theorem 1, pg. 152 in [1]) consists in:

The Riemann Hypothesis holds if and only if $\mathrm{T}_{\varphi}$ defined on $\mathrm{L}^{2}(0,1)$ by

(4) $\quad\left(\mathrm{T}_{\varphi} \mathrm{u}\right)(\mathrm{y})=\int_{0}^{1}\{y / x\} u(x) d x$ is injective.

Applying the criteria described for integral operators taking the dense family $\chi$ we obtain the entries of the diagonal matrix representations of restrictions of $\mathrm{T}_{\varphi}$ that, are all strict positive valued ([5], [6]):

$$
a_{11}^{h}=\frac{h^{2}}{4}(3-2 \gamma) ; a_{k k}^{h}=\frac{h^{2}}{2}\left(-1+\frac{2 k-1}{k-1} \ln \left(\frac{k}{k-1}\right)^{k-1}\right), \mathrm{k}=2, \mathrm{n}, \mathrm{nh}=1,
$$

where $\gamma$ is the Euler-Mascheroni constant, falling between first and the second diagonal entries. Thus, $\mathrm{T}_{\varphi}$ is strict positive definite on each subspace, i.e. $\mathrm{T}_{\varphi} \in L_{\chi}$. Similar evaluations we obtained for the adjoint restrictions (see [5] v8 for optimal values) and, as result the condition numbers sequence is inferior bounded by a constant $([5],[6])$ :

(5) $\quad \mu_{h}\left(\mathrm{~T}_{\varphi}\right) \geq(3-2 \gamma) / 2(3 \ln 2-1)>0, \mathrm{nh}=1, \mathrm{n} \geq 2$. Thus, follows:

Theorem 2. The integral operator $\left(\mathrm{T}_{\varphi} \mathrm{u}\right)(\mathrm{y})=\int_{0}^{1}\{y / x\} u(x) d x$ defined on $\mathrm{L}^{2}(0,1)$ is injective.

So, we could claim: Riemann Hypothesis holds.

5. Observations. The integral operator $\mathrm{T}_{\{y / x\}}$ has been introduced by Beurling in 1956 ([2]) for providing an equivalent formulation of $\mathrm{RH}$ (1859), in terms of functional analysis. In 1992, using the Beurling formulation Alcantara-Bode provided another formulation of $\mathrm{RH}([1])$ in terms of the injectivity of an integral operator that has been proved in [5] and [6].

Now, no tentative has been successful in providing a solution to $\mathrm{RH}$ following Beurling equivalent formulation of RH ([2]), nor following AlcantaraBode equivalent formulation ([1]). However, their outstanding equivalences of RH taking RH outside the pure mathematics world, allowed us to provide a solution to $\mathrm{RH}$ addressing the Alcantara-Bode equivalent formulation by means of applied mathematics techniques. 


\section{References}

[1] Alcantara-Bode, J., (1993) "An Integral Equation Formulation of the Riemann Hypothesis", Integr. Equat. Oper. Th, Vol. 17, 1993.

[2] Beurling, A., (1955) "A closure problem related to the Riemann zeta function", Proc. Nat. Acad. Sci. 41 pg. 312-314, 1955.

[3] Buescu, J., Paixa $\sim$ o A. C., (2007) "Eigenvalue distribution of Mercer-like kernels", Math.Nachr.280, No.9-10, pg. 984 - 995, 2007.

[4] Rudin, W., "Real and Complex Analysis", McGraw-Hill International, Third Edition 1987

[5] Adam, D., (2021) DOI:10.21203/rs.3.rs-1159792/v8, preprint, Research Square, Dec. 2021.

[6] Adam, D., (2022) "On the Injectivity of an Integral Operator Connected to Riemann Hypothesis", J. of Pure and Appl. Math., Vol.6, Issue 4 (in printing process).

[7] Adam, D., (1994), "Mesh Independence of Galerkin Approach by Preconditioning", in "Preconditioned iterative methods", JH Libraries, Lausanne, Switzerland; [Langhome, Pa.]: Gordon and Breach, 1994

[8] Balazard M., Saias E., (2000) "The Nyman-Beurling equivalent form for the Riemann hypothesis", Expo. Math. 18, 131-138 (2000)

[9] Bompieri, E., (2000) "www.claymath.org/millennium-problems/riemannhypothesis", Problems of the Millennium:

the Riemann Hypothesis, Clay Institute annual report, 2000.

[10] Chang, C.H., Ha, C.W. (1999) "On eigenvalues of differentiable positive definite kernels", Integr. Equ. Oper. Theory 33 pg. 1-7, 1999.

[11] Fan, K. (1954) "Inequalities for eigenvalues of Hermitian matrices", Nat. Bur. Standards Appl. Math. Ser. 39 pg. 131-139, 1954.

[12] Kolotilina, L. Yu. (1995) "Interrelations between eigenvalues and diagonal entries of Hermitian matrices implying their block diagonality", Zap. Nauchn. Sem. POMI, 1995, $153-158$

[13] Mercer, J., (1909) "Functions of positive and negative type and their connection with the theory of integral equations", Philosophical Transactions of the Royal Society A 1909

[14] Papadopoulos, P., "https://csml.berkeley.edu/Notes/ME280A.pdf", 2015

[15] Reade, J.P., (1983) "Eigenvalues Of Positive Definite Kernels", SlAM J. Math. Anal. Vol. 14, No January 1983. 